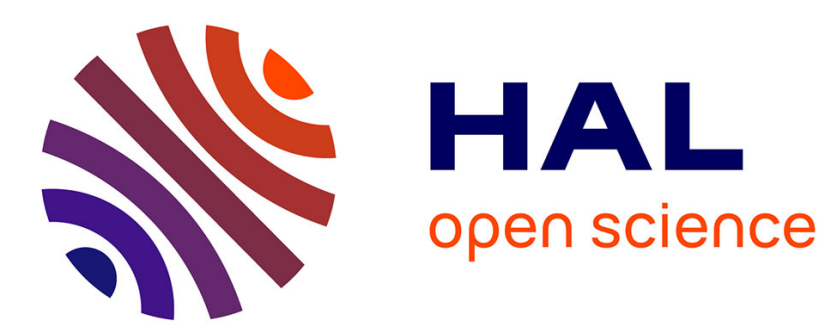

\title{
Synchrotron X-ray diffraction study of the room temperature incommensurate phase in graphite-bromine intercalation compound
}

\author{
D. Ghosh, D.D.L. Chung
}

\section{- To cite this version:}

D. Ghosh, D.D.L. Chung. Synchrotron X-ray diffraction study of the room temperature incommensurate phase in graphite-bromine intercalation compound. Journal de Physique Lettres, 1983, 44 (18), pp.761-769. 10.1051/jphyslet:019830044018076100 . jpa-00232260

HAL Id: jpa-00232260

https://hal.science/jpa-00232260

Submitted on 1 Jan 1983

HAL is a multi-disciplinary open access archive for the deposit and dissemination of scientific research documents, whether they are published or not. The documents may come from teaching and research institutions in France or abroad, or from public or private research centers.
L'archive ouverte pluridisciplinaire HAL, est destinée au dépôt et à la diffusion de documents scientifiques de niveau recherche, publiés ou non, émanant des établissements d'enseignement et de recherche français ou étrangers, des laboratoires publics ou privés. 
Classification

Physics Abstracts

$61.50 \mathrm{~K}$

\title{
Synchrotron $X$-ray diffraction study of the room temperature incommensurate phase in graphite-bromine intercalation compound $(*)$
}

\author{
D. Ghosh and D.D.L. Chung \\ Department of Metallurgical Engineering and Materials Science, Carnegie-Mellon University, \\ Pittsburgh, PA 15213, U.S.A.
}

(Reçu le 25 avril 1983, accepté le 20 juillet 1983)

\begin{abstract}
Résumé. - On a utilisé des rayons $\mathrm{X}$ émis par un synchrotron en coordination avec un diffractomètre à quatre cercles dans le but d'étudier la phase incommensurable dans un composé graphitebromine en second stade à température ambiante. Le composé graphite-brome a été préparé à partir d'un cristal uniqu. de graphite. Les réflexions de super réseau les plus fortes et les satellites les plus importants ont été balayés le long de la direction la plus incommensurable du réseau entre $302 \mathrm{~K}$ et $324 \mathrm{~K}$. La transition incommensurable-commensurable qui a lieu à $319 \pm 1 \mathrm{~K}$ produit la disparition des pics incommensurables et l'apparition du pic commensurable. Nous présentons des modèles possibles pour la phase incommensurable.
\end{abstract}

\begin{abstract}
Synchrotron X-ray and a four-circle diffractometer were used to study the room temperature incommensurate phase in stage- 2 graphite-bromine prepared with single crystal graphite in bromine liquid. The two strongest in-plane superlattice reflections and the strongest satellite peak were scanned along the most incommensurate direction of the lattice between $302 \mathrm{~K}$ and $324 \mathrm{~K}$. The incommensurate-commensurate transition at $319 \pm 1 \mathrm{~K}$ caused disappearance of the incommensurate peaks and appearance of the commensurate one. Possible models of the incommensurate phase are presented.
\end{abstract}

The commensurate-incommensurate (C-I) transition has lately become a well established phenomenon in two-dimensional as well as three-dimensional systems. Along with the development of the theory [1, 2], X-ray and neutron scattering experiments have provided the basis for the understanding of this transition. Two of the systems where this topic was investigated in detail are a monolayer of $\mathrm{Kr}$ adsorbed on graphite and the dichalcogenides (namely $2 \mathrm{H}-\mathrm{TaSe}_{2}$ and $\left.2 \mathrm{H}-\mathrm{NbSe}_{2}\right)[3,4]$. Recent theories have attempted to interpret the diffraction results in terms of domain wall and « soliton " models $[5,6]$. Low energy electron diffraction experiments have also provided the evidence of this transition [ 7].

(*) Research sponsored by the Ceramics Program, Division of Materials Research, National Science Foundation, Grant No. DMR-7926242. 
Graphite-bromine intercalation compounds, a less studied system than many other intercalation compounds, is known to exhibit quasi two-dimensional character because of the lack of correlation among various intercalate layers $[8,9]$. However, the in-plane structure of the bromine atoms is well defined. The structure of the stage- 2 commensurate phase (Type A) studied by X-ray diffraction has been shown to possess polybromide chains $[8,9]$. The first evidence of a transition in a desorbed HOPG-bromine system came from an in-plane X-ray diffraction experiment [10]. Following investigations by X-ray and Raman scattering revealed the details of this transition and also the stage dependence of the transition temperature [11-13].

We recently reported the existence of a room temperature incommensurate (I) phase in " type B " graphite-bromine (i.e., stage-2, prepared with graphite single crystals in liquid bromine and maintained in contact with liquid bromine) [12]. This phase was also observed by others [8, 14], although its incommensurate diffraction pattern had not been interpreted. From the precession photographic recording of the in-plane diffraction pattern, we showed that, at $316 \mathrm{~K}$, type $\mathrm{B}$ exhibit a reversible phase transition from the incommensurate structure characteristic of type $B$ at room temperature to the commensutate structure characteristic of type A (stage-2, prepared in bromine vapour) at room temperature [9]. We termed this transition an incommensuratecommensurate (I-C) transition [12], which is distinct from the C-I transition.

Two distinctive features of the I phase were noted. Firstly, the 3,0 reflection, the strongest inplane superlattice reflection in the commensurate $(C)$ phase $\left(a=8.87 \AA, b=4.26 \AA, \gamma=103.9^{\circ}\right.$; primitive monoclinic cell [15]; Fig. 1), occurred at a higher $Q$ value in the I phase. Secondly, diffused lines weakly present in the $\mathrm{C}$ phase developed strongly in the I phase at $h, k(k \neq 0)$ positions parallel to the $a^{*}$-direction. These observations suggested that the bromine chains in the I phase are periodically spaced along the $a$-direction, while the atom-to-atom correlation along the $b$-direction among the chains is limited. The situation is thus quasi one-dimensional. In the present X-ray diffraction experiment, the intensity scan was performed along this direction of quasi one-dimensional periodicity, i.e., the direction perpendicular to the length of the polybromide chains, namely the $a^{*}$-axis. Strictly speaking, the diffraction pattern [12] suggests that the

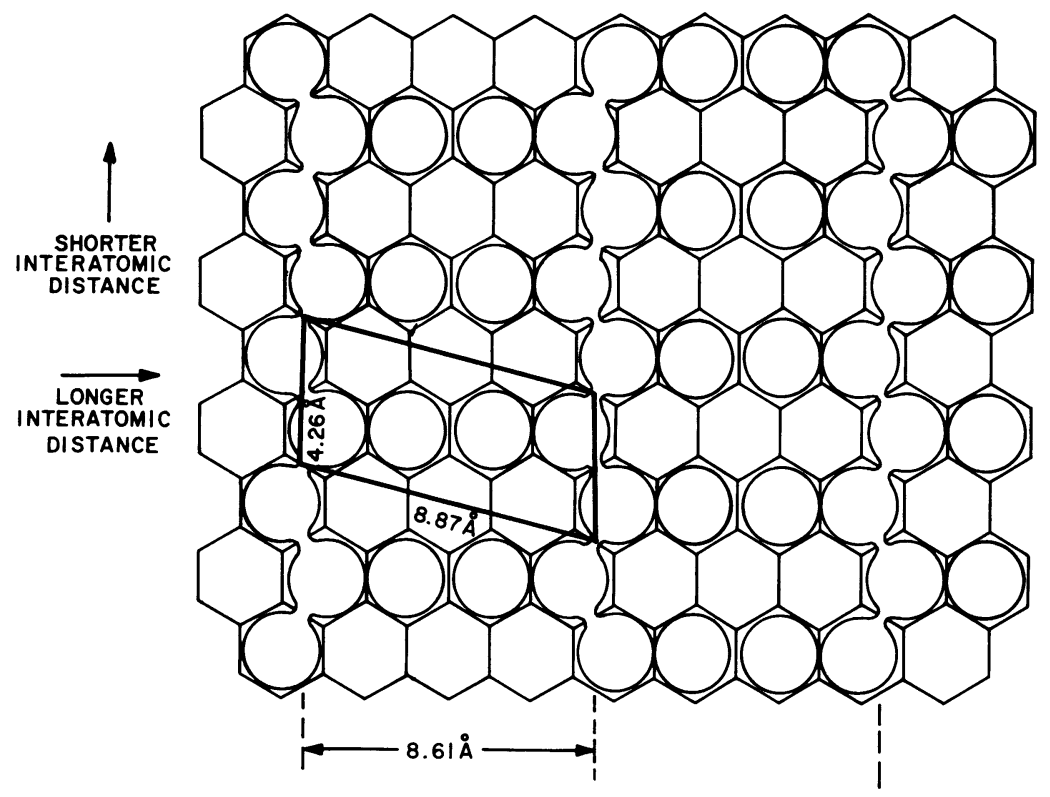

Fig. 1. - In-plane crystal structure of the $\mathrm{C}$ phase. The bromine chains are in the direction corresponding to the smallest bromine-bromine interatomic distance. The parallelogram is the primitive unit cell. 
in-plane structure has some correlation along the $b^{*}$-direction also and one has to perform scans along the diffuse lines to obtain a complete account of this structural transition. Nonetheless, the present one-dimensional study is significant in that the scan is performed along the most incommensurate and the most correlated direction.

The diffraction experiment was performed at the Cornell High Energy Synchrotron Source (CHESS) on a modified Picker four-circle X-ray diffractometer. The crystal, $\sim 1.0 \mathrm{~mm}$ in diameter, was sealed with liquid bromine in a cylindrical pyrex glass capillary and was mounted on the diffractometer with the $c$-axis along the beam direction. The beam was tuned to $9.731 \pm$ $0.002 \mathrm{keV}$. The photon flux was $\sim 10^{10}$ photons $/ \mathrm{s}$ on the crystal, at $\sim 5 \mathrm{GeV}$ and $15 \mathrm{~mA}$. A $\omega / 2 \theta$ step scan was used; the step size was 0.01667 degree. Taking the HWHM of the graphite $h, k$ peaks as the resolution, the $Q$ resolution was $0.01 \AA^{-1}$. However, the actual resolution was better than this value because of the inherent broadening in the graphite peaks. The FWHM of the 3,0 superlattice peak was $0.03 \AA^{-1}$ with synchrotron X-rays, as compared to $0.18 \AA^{-1}$ with fine-focus $\mathrm{MoK} \alpha$ radiation. The improvement in the signal-to-noise ratio for the strong superlattice reflections was about an order of magnitude over the standard $1 \mathrm{KW}$ fine-focus tube setup.

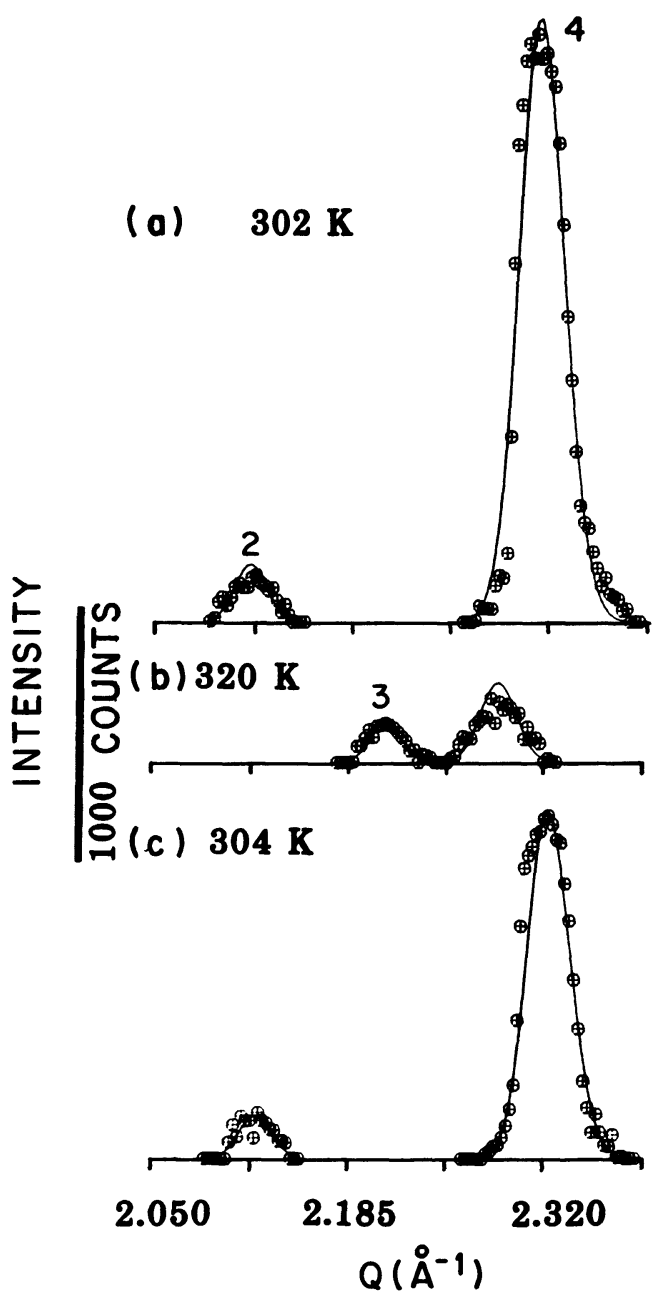

Fig. 2. - Scan of intensity vs. $Q$ for type B along $\mathrm{a}^{*}$ in the vicinity of 3,0 of the $\mathrm{C}$ phase. The peaks are labelled as 2, 3 and 4 . 
The crystal was surrounded by a microheater (Enraf-Nonius FR 559) mounted on the goniometer head and was heated by the power supplied through a temperature controller. A second thermocouple was mounted on the outer wall of the glass capillary next to the crystal. The maximum error in the temperatures measured was about $\pm 1 \mathrm{~K}$.

Scans were performed on type $B$ at various temperatures in the neighbourhood of the two strongest superlattice reflections, close to $2,0\left(Q=1.460 \mathrm{~A}^{-1}\right)$ and $3,0\left(Q=2.189 \AA^{-1}\right)$ of the $\mathrm{C}$ phase. Figure $2 a$, shows the scan for type $\mathrm{B}$ at $302 \mathrm{~K}$ in a region of $Q$ close to 3,0 of the $\mathrm{C}$ phase. The peak had shifted up to $Q=2.317 \pm 0.002 \AA^{-1}$ along $a^{*}$ (Peak 4). In addition, a satellite peak appeared at $Q=2.116 \pm 0.003 \AA^{-1}$ (Peak 2$)$. This is the strongest satellite peak, as indicated by the rotating anode photograph (Fig. 5 of Ref. 14). Although there was no noticeable change in the position of peak 2 between $302 \mathrm{~K}$ and $316 \mathrm{~K}$, peak 4 continuously shifted down in $Q$ upon heating. However, peak 4 remained significantly above the commensurate value; the maximum shift was only $\sim 1 \%$ near the transition. At $317 \mathrm{~K}$, there was a drastic reduction in the intensities of both peaks 2 and 4. Beyond this temperature, the integrated intensities of these two peaks continuously decreased and fell to the background level at $\sim 323 \mathrm{~K}$ (Fig. 3). At about $317 \mathrm{~K}$, a new peak (Peak 3) appeared at the commensurate $Q$ value of the 3,0 peak, namely at $2.205 \pm$ $0.009 \AA^{-1}$ (Fig. $2 b$ ). The integrated intensity of peak 3 (Fig. 3) reached its maximum at $\sim 320 \mathrm{~K}$ and stayed there above $320 \mathrm{~K}$.

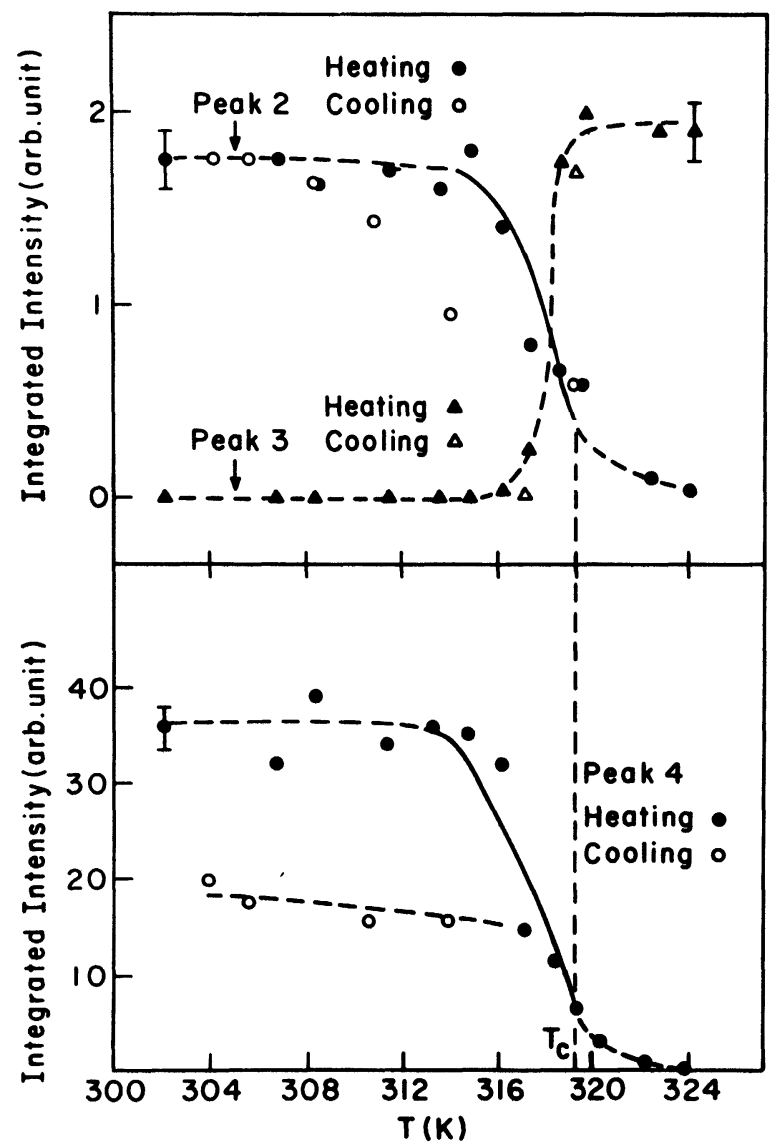

Fig. 3. - Integrated intensity (in an arbitrary unit) $v s$. temperature $T$ for peaks 2 and 3 (upper panel) and peak 4 (lower panel) during heating (solid symbols) and cooling (open symbols). The solid curves were obtained by least-squares fitting to a power law. 


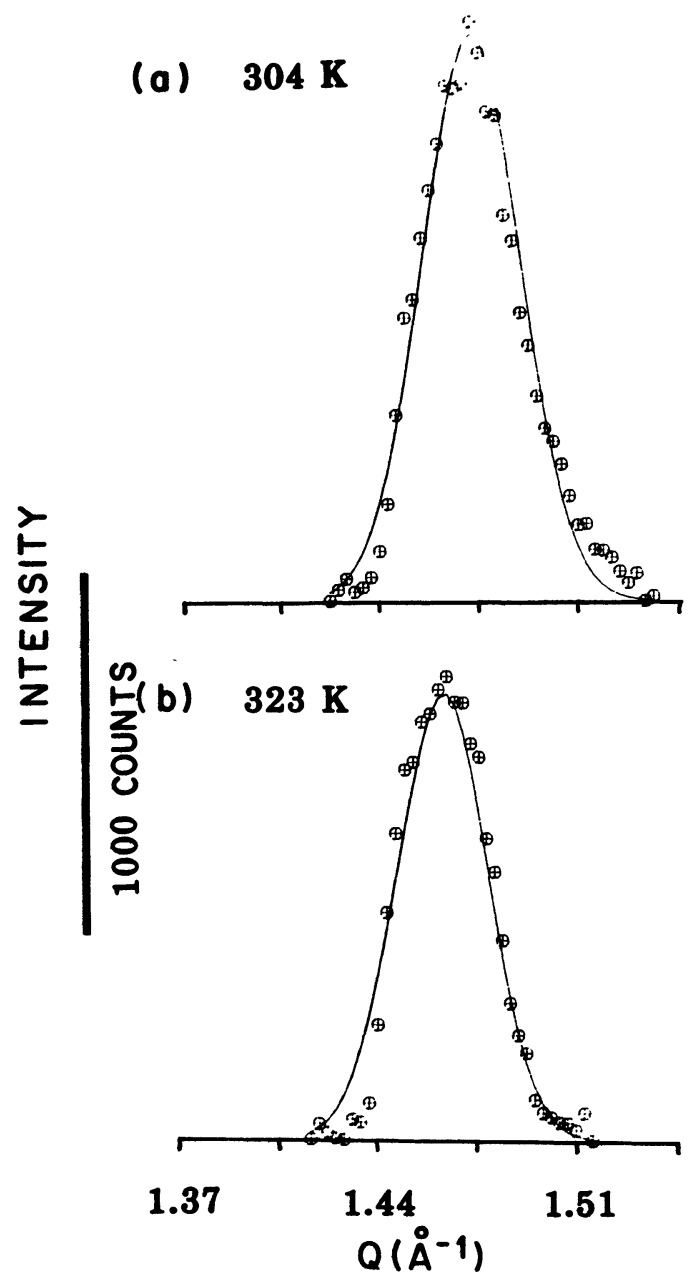

Fig. 4. - Scan of intensity $v s$. $Q$ for type B along $\mathrm{a}^{*}$ in the vicinity of 2,0 of the $\mathrm{C}$ phase, showing peak 1 .

Figure $4 a$ shows the scan for type B at $304 \mathrm{~K}$ in a region of $Q$ close to 2,0 of the $\mathrm{C}$ phase. The 2,0 peak had shifted up to $Q=1.475 \pm 0.003 \AA^{-1}$ (Peak 1). As the temperature was raised, peak 1 continuously shifted down in $Q$ (Fig. 4, Fig. 5); at $\sim 319 \mathrm{~K}$, it assumed, within the limits of experimental error, the 2,0 commensurate value of $1.460 \AA^{-1}$. The FWHM of this peak also decreased with increasing temperature (Fig. 5). In addition, the integrated intensity of peak 1 decreased by about $20 \%$ (Fig. 4).

On subsequent cooling from $323 \mathrm{~K}$ to room temperature, the I phase was again obtained (Fig. 2c, Fig. 3). Thus, the transition appeared to be reversible. Although the intensities of peaks 2 and 3 returned to their initial values after cooling, that of peak 4 was not completely recovered (Fig. 3). This was probably due to intercalate desorption, even though the sample was sealed with liquid bromine in a capillary. As a result, the existence of hysteresis cannot be ruled out. The I phase is probably more concentrated than the $\mathbf{C}$ phase. Consequently, during the I-C transition, desorption of bromine and reabsorption during the reverse process might take place.

In figures 2 and 4, a linear background had been subtracted from the scans and the peaks were fitted to a Gaussian profile by the least-squares technique. Fitting to a Lorentzian profile was also tried, but a Gaussian function alone was found to describe the peaks most satisfactorily. 


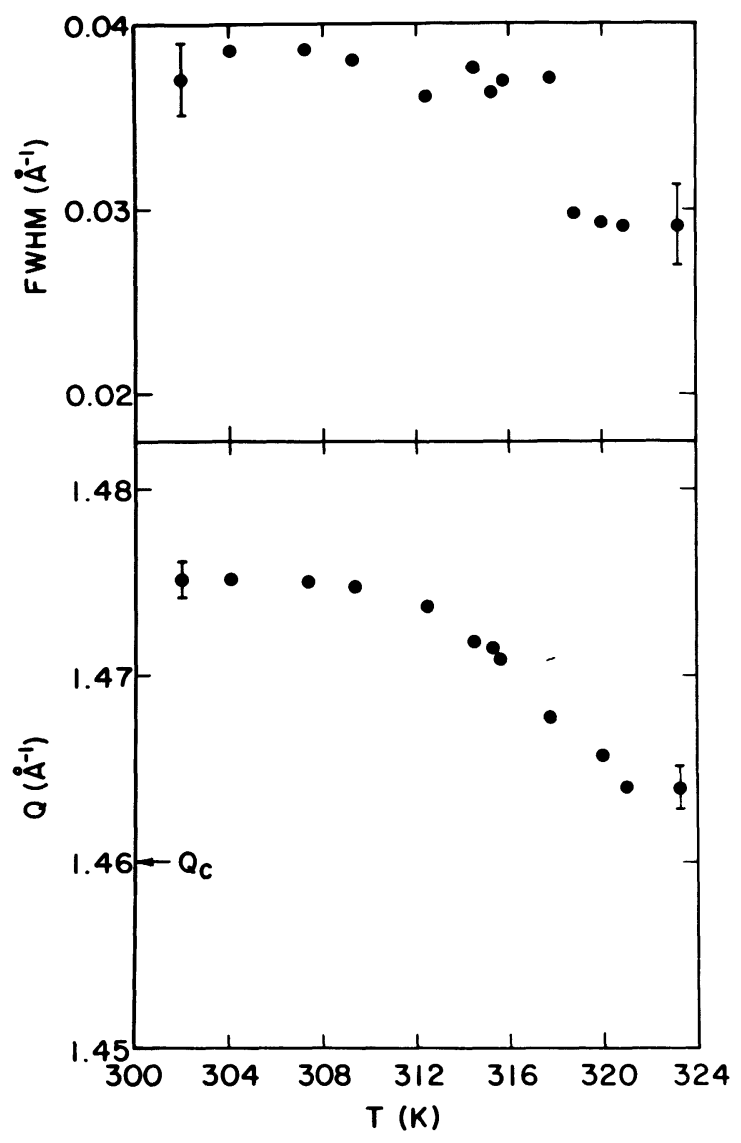

Fig. 5. - FWHM and $Q$ vs. $T$ for peak 1 during heating.

The presence of a sufficient number of data points over the peak region also enabled us to calculate the parameters numerically and compare with the fitted values. The broadening on the high $Q$ side, originating from the mosaic tilts of the Bragg rods, was also noticed for some peaks. However, this effect was negligibly small and never seriously affected our symmetric profile fitting. Nonetheless, there could be some ambiguity regarding the exact shape of the profiles, but our results are independent of any particular shape.

These results clearly indicate a transition from the I phase to the $C$ phase at about $319 \pm 1 \mathrm{~K}$ upon heating. This is in good agreement with our previous photographic result $\left(T_{1}=320 \mathrm{~K}\right)[12]$. Since both peaks 1 and 4 continuously shifted down in $Q$ upon heating, the incommensuration $\varepsilon$ seemed to be a function of temperature. In the photographic experiment [12], because of the lack of sufficient resolution, an average effect of all the observations described above was noticed. This effect was interpreted as the shifting of the incommensurate peak 4 to the commensurate 3,0 position [12]. In the photographic results, the satellite peak (Peak 2) was unresolved from the primary (Peak 4). The shift in the position of peak 1 to that of the commensurate phase also could not be ascertained by the photographic method.

Let us turn our attention to the geometrical aspects of the positions of the peaks and their relevance to the real lattice. The behaviour of peaks 1 and 4 appeared quite dissimilar; peak 1 at higher temperatures shifted to the commensurate position, while the I-C transition for peak 4 was characterized mainly by the disappearance of the incommensurate peak and the appearance 
of a new peak at the commensurate position. The possibility of these two peaks belonging to two separate incommensurate phases is unlikely since, among other reasons to be discussed, both conform to the same I-C transition temperature. The incommensurate phase reported here, in its relation to the corresponding commensurate phase, is quite different from those previously reported, namely $\mathrm{Kr}$ on graphite and high temperature graphite-bromine. The difference is primarily in two respects. (i) It is very strongly incommensurate, in which intra-intercalate interaction possibly predominates over intercalate-host interaction. The diffraction primaries are appreciably shifted from the commensurate positions, all to the higher $Q$ side. Secondary or satellite peaks appear for some reflections in positions such that all the peaks along the $a^{*}$-axis (Fig. 5 of Ref. 14) can be indexed with integral Miller indices using a fairly short unit cell, $29.8 \pm$ $0.1 \AA$. Thus, in contrast to the classical domain-wall model of Frank and van der Merwe [7], where incommensuration is due to the creation of domain walls while the atoms are in registry with the host lattice, we may really have here unregistered chains of atoms in the domain. (ii) The I-C transition is characterized by the disappearance of the incommensurate peaks, peaks 2 and 4 at $Q_{\mathrm{c}}(1-0.033)$ and $Q_{\mathrm{c}}(1+0.058)$, respectively, and the appearance of the commensurate peak, peak 3 , in contrast to the behaviour of the peaks in the C-I transitions previously studied.

Nonetheless, our data cannot be interpreted on the basis of a continuum incommensurate model. The interchain distance in the $\mathrm{C}$ phase is $8.61 \AA$, which is half the orthorhombic repeat length of $17.22 \AA$. Since the interchain distance cannot be drastically reduced in the continuum incommensurate model, a unit cell $29.8 \AA$ long cannot contain an integral number of evenly spaced chains. Thus, inhomogeneity or « domain wall » has to be introduced to explain the peak positions. These walls should be separated from each other by $29.8 \pm 0.1 \AA$.

We have carried out detailed calculations for various models involving different domain wall thicknesses and domain wall separations. In particular, a commensurate domain wall thickness of multiples of $2.46 \AA$ and very large domain wall separations, like those in other systems [3,11] could not explain our data satisfactorily. In a simple one-dimensional situation, the intensity of a peak at position $Q$ for a finite coherence length is given by

$$
I \sim K \frac{\sin ^{2} \frac{1}{2} Q n d}{\sin ^{2} \frac{1}{2} Q d} \frac{\sin ^{2} \frac{1}{2} Q N D}{\sin ^{2} \frac{1}{2} Q D} .
$$

$K$ is the (amplitude) $)^{2}$ for a single basic unit of length $d . D=n d+\delta$, with $\delta$ being the thickness of a domain wall. In equation 1 , the scattering due to domain walls has been ignored. Only two models were found to give satisfactory fits to the observed diffraction pattern. In these models, $D=29.72 \pm 0.03 \AA$. In the first model, model $\mathrm{I}$, with domains having Marti and Thorel type unregistry [16], the interchain distance is taken as $3 \times 2.711 \AA=8.133 \AA$, where $2.711 \AA$ is the $d$ value of peak 4 . Hence, $D=3 \times 8.133+5.32=29.72 \AA$. For $N>25$ the calculated positions and relative intensities of peaks 1,2 and 4 agree reasonably well with the observed values. These results are shown in table I. In the second model, model II, the domains are in Frank and van der

Table I. - Observed and calculated peak positions and intensities.

\begin{tabular}{|c|c|c|c|c|c|}
\hline Peak No. & \multirow{2}{*}{$\begin{array}{c}\text { Observed } Q \\
\left(\AA^{-1}\right)\end{array}$} & $\begin{array}{c}\text { Calculated } Q \\
\left(\AA^{-1}\right)\end{array}$ & $\begin{array}{c}\text { Observed intensity } \\
\text { (arbitrary unit) }\end{array}$ & \multicolumn{2}{|c|}{ Calculated intensity } \\
\cline { 5 - 6 } & $1.475 \pm 0.003$ & $1.477 \pm 0.002$ & 173 & 166 & 400 \\
\hline 1 & $2.116 \pm 0.003$ & $2.118 \pm 0.002$ & 13 & 16 & 13 \\
4 & $2.317 \pm 0.002$ & $2.323 \pm 0.002$ & 200 & 200 & 200 \\
\hline
\end{tabular}


Merwe type registry, with incommensurate domain walls. The wall-to-wall length is thus given by $D=3 \times 8.61+3.89=29.72 \AA$. The relative intensities of peaks 2 and 4 calculated from this model agree quite well with the observed values for $N>121$. However, the calculated intensity of peak 1 is appreciably higher.

Both models predict higher order primary and satellite peaks to be observed at $Q$ values of 1.68 , $2.54,2.95,3.17,3.38,3.59$ and $3.79 \AA^{-1}$. Indeed, these peaks account for the rest of the peaks present in the diffraction photograph (Fig. 5 of Ref. 14). In addition, model I shows a peak at $Q=$ $1.54 \AA^{-1}$, which is unobserved experimentally. In our calculation, however, we find that this peak is extremely weak for certain ranges of $N$, e.g., near $N=44$. Calculation based on model II predicts one more peak at $2.75 \AA^{-1}$, which is unobserved.

Our calculations suggest that the coherence length is not infinitely large, but is limited to finite values in both the models. This finding in samples prepared with single crystals of graphite might be indicative of the formation of islands of three possible in-plane orientations [15].

The intensity data at temperature $T$ near the transition temperatures $T_{\mathrm{c}}$ were least-squares fitted to a power law, as indicated by solid lines in figure 3. It was assumed that the total diffracted intensities $I$ for peaks 2 and 4 were given by $I \propto\left(T_{\mathrm{c}}-T\right)^{2 \beta}$, where $T_{\mathrm{c}}=319 \mathrm{~K}$. The values of the critical exponent $\beta$ derived from peaks 2 and 4 were $0.24 \pm 0.05$ and $0.25 \pm 0.05$, respectively. These values are comparable to that of 0.32 reported on the melting in graphite-bromine at $374 \mathrm{~K}$ [10]. This similarity is consistent with the fact that both the I-C transition and the melting involve the gradual disappearance of a phase. In contrast, the C-I transition involves the shifting of peaks rather than the disappearance of peaks. The intensity seems to tail off to zero beyond $T_{\mathrm{c}}$. This is probably due to the variation in the critical temperature arising from the 2-D finite sizeeffect [17].

Finally, in conclusion, we have demonstrated that at about $319 \mathrm{~K}$ there is a structural change in the incommensurate graphite-bromine phase, in which the inter-chain distance as well as the " domain » structure are modified to form the commensurate phase. The present one-dimensional analysis is preliminary and the diffuse lines are presently being studied.

\section{Acknowledgments.}

The authors thank CHESS staff members for their help during data collection, R. Clarke for providing the single crystal graphite material and $\mathbf{R}$. Moret for providing a copy of his rotating anode photograph. Discussions with R. M. Suter and S. H. Anderson have been very helpful. R. Gangwar and B. Manley are thanked for technical assistance.

\section{References}

[1] Nattermann, T., Frimper, S., J. Physique 43 (1982) 23.

[2] Coppersmith, S. N., Fisher, D. S., Halperin, B. I., Lee, P. A., Brinkman, W. F., Phys. Rev. B 25 (1982) 349.

[3] Stephens, P. W., Heiney, P., Birgeneau, R. J.,Horn, P. M., Phys. Rev. Lett. 43 (1979) 47.

[4] Moncton, D. E., AXe, J. D., DiSalvo, F. J., Phys. Rev. B 16 (1977) 801.

[5] Nielsen, M., Als-Nielsen, J., McTague, J. P., in Ordering in Two Dimensions, edited by S. K. Sinha (North-Holland) 1980 , p. 135.

[6] DoRner, B., in Structural Phase Transitions I, edited by K. A. Müller and H. Thomas (Springer-Verlag) 1981, p. 93, and references therein.

[7] Frank, F. C., van der Merwe, J. H., Proc. R. Soc. A 198 (1949) 205, 216.

[8] Eeles, W. T., Turneull, J. A., Proc. R. Soc. A 283 (1965) 179.

[9] Ghosh, D., Chung, D. D. L., Mater. Res. Bull. 18 (1983), in press. 
[10] Bardhan, K. K., Wu, J. C., Chung, D. D. L., Synth. Met. 2 (1980) 109.

[11] Kortan, A. R., Erbil, A., Birgeneau, R. J., Dresselhaus, M. S., Phys. Rev. Lett. 49 (1982) 1427.

[12] Ghosh, D., Chung, D. D. L., Mater. Res. Soc. Symp. Proc., Vol. 20 (Elsevier) 1983, p. 15-20.

[13] Erbil, A., Dresselhaus, G., Dresselhaus, M. S., Phys. Rev. B 25 (1982) 5451.

[14] Moret, R., Comes, R., Furdin, G., Fuzellier, H., Rousseaux, F., Proceedings of Symposium on Intercalated Graphite, Annual Meeting of Materials Research Society, Boston, 1982, Ed. M. S. Dresselhaus (Elsevier).

[15] Ghosh, D., Chung, D. D. L., Mater. Res. Bull. 18 (1983) 727.

[16] Marti, C., Thorel, P., J. Physique Colloq. 38 (1977) C4-26.

[17] Horn, P. M., Birgeneau, R. J., Heiney, P., Hammons, E. M., Phys. Rev. Lett. 41 (1978) 961. 